\title{
Using Volunteered Geographic Information (VGI) in Design-Based Statistical Inference for Area Estimation and Accuracy Assessment of Land Cover
}

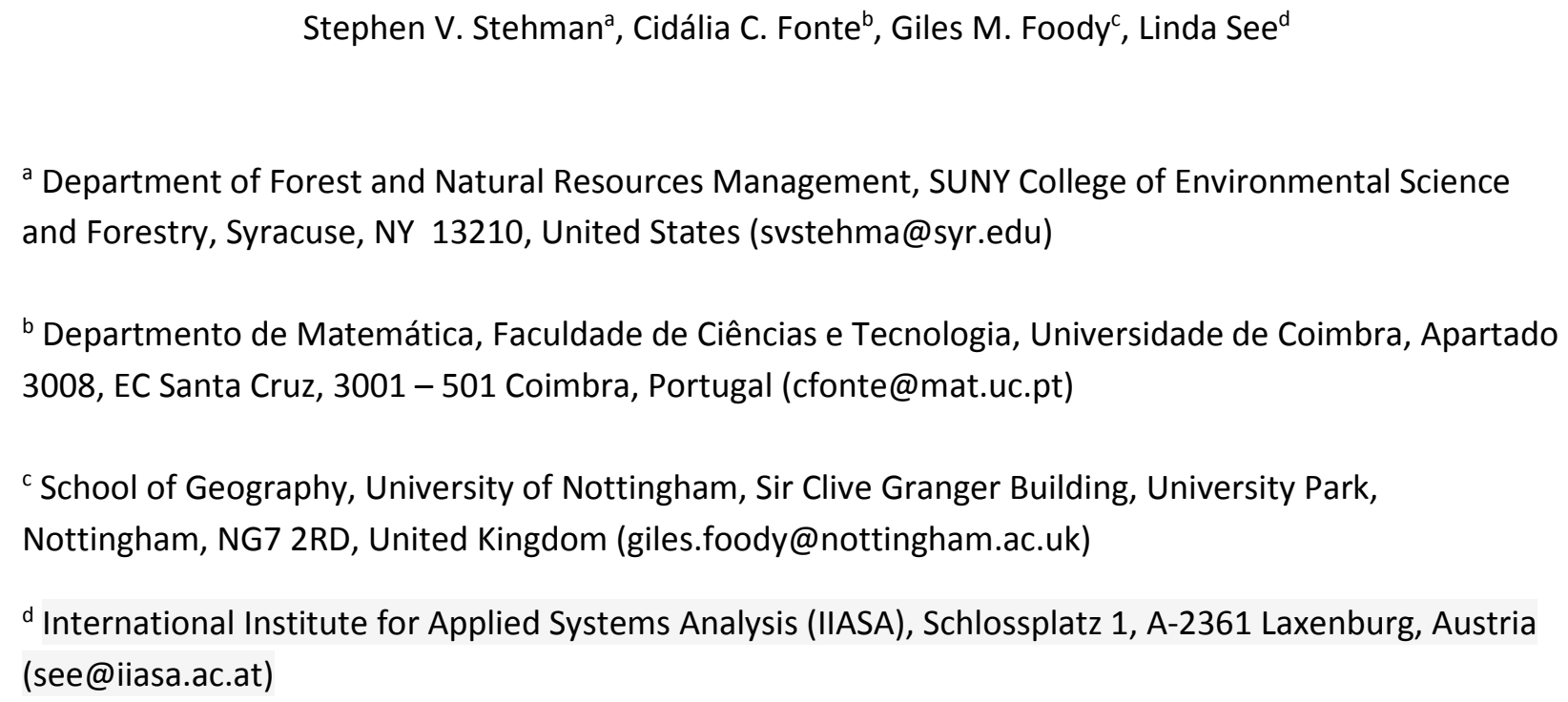

\section{Abstract}

Volunteered Geographic Information (VGI) offers a potentially inexpensive source of reference data for estimating area and assessing map accuracy in the context of remote-sensing based land-cover monitoring. The quality of observations from VGI and the typical lack of an underlying probability sampling design raise concerns regarding use of VGI in widely-applied design-based statistical inference. This article focuses on the fundamental issue of sampling design used to acquire VGI. Design-based inference requires the sample data to be obtained via a probability sampling design. Options for incorporating VGI within design-based inference include: 1) directing volunteers to obtain data for locations selected by a probability sampling design; 2) treating VGI data as a "certainty stratum" and augmenting the VGI with data obtained from a probability sample; and 3) using VGI to create an auxiliary variable that is then used in a model-assisted estimator to reduce the standard error of an estimate produced from a probability sample. The latter two options can be implemented using VGI 
32 data that were obtained from a non-probability sampling design, but require additional sample data to

33 be acquired via a probability sampling design. If the only data available are VGI obtained from a non-

34 probability sample, properties of design-based inference that are ensured by probability sampling must

35 be replaced by assumptions that may be difficult to verify. For example, pseudo-estimation weights can

36 be constructed that mimic weights used in stratified sampling estimators. However, accuracy and area

37 estimates produced using these pseudo-weights still require the VGI data to be representative of the full

38 population, a property known as "external validity". Because design-based inference requires a

39 probability sampling design, directing volunteers to locations specified by a probability sampling design

40 is the most straightforward option for use of VGI in design-based inference. Combining VGI from a non-

41 probability sample with data from a probability sample using the certainty stratum approach or the

42 model-assisted approach are viable alternatives that meet the conditions required for design-based

43 inference and use the VGI data to advantage to reduce standard errors.

Key Words: probability sampling; external validity; pseudo-weights; data quality; model-based

46 inference; Volunteered Geographic Information (VGI); crowdsourcing

\section{Introduction}

Volunteered Geographic Information (VGI) is defined as "tools to create, assemble, and

50 disseminate geographic data provided voluntarily by individuals" (Goodchild 2007). For land-cover

51 studies, VGI may provide the reference condition or the information used to determine the reference

52 condition of a spatial unit. The reference condition, defined as the best available assessment of the

53 ground condition, plays a critical role in accuracy assessment and area estimation (Olofsson et al. 2014).

54 When used in map production, VGI could form all or part of the data used to train the land-cover

55 classification algorithm. The focus of this article is the contribution of VGI to the reference data used for 
accuracy assessment and area estimation. Accuracy assessment is an essential component of a rigorous

57 mapping-based analysis of remotely sensed data as without it the obtained products are little more than pretty pictures and simply untested hypotheses (McRoberts 2011; Strahler et al. 2006). In addition an accuracy assessment adds value to a study, especially when estimates of class area (e.g. deforestation) are to be obtained (Olofsson et al. 2014). Fonte et al. (2015) examined the use of VGI for land cover validation, including the types of VGI that have been used, the main issues surrounding VGI quality assessment, and examples of VGI projects that have collected data for validation purposes. We build upon this past work to focus on the issue of statistical inference when incorporating VGI in applications of accuracy and area estimation, but our work is also relevant to application of citizen science data in general (Bird et al. 2014). reference condition on a per spatial unit basis (e.g., pixel, block, or segment). Accuracy assessment typically focuses on producing an error matrix and associated summary measures including overall, user's, and producer's accuracies (see Section 2 for details). Estimates of area of each land-cover class or type of land-cover change based on the reference condition are often produced in conjunction with

71 the accuracy estimates (Olofsson et al. 2013, 2014). Sampling, defined as selecting a subset of the

72 population, is almost always necessary because it is too costly to obtain a census of the reference 73 condition. VGI represents a subset of the population and as such may be viewed as a sample. Whether the VGI data were collected via a probability sampling design is a key consideration when evaluating the utility of VGI for design-based inference. Design-based inference is a standard, widely used approach adopted in environmental science for furthering knowledge and understanding on the basis of a sample 77 of cases rather than a study of the entire population. We describe options for incorporating VGI into map accuracy assessment and area estimation 
VGI can be transformed into more precise estimators (i.e., smaller standard errors, a desirable outcome

81 of an effective sampling strategy) within the scientifically defensible framework provided by design-

82 based inference. If the VGI data are obtained via a probability sampling design, application of design-

83 based inference is straightforward and can be informed by good practice guidelines (Olofsson et al.

84 2014). Alternatively, if the VGI data are not obtained via a probability sampling protocol, the VGI data

85 can be combined with additional data from a probability sample to produce estimates that satisfy the

86 conditions underlying design-based inference. In such cases the VGI data from a non-probability sample

87 serve as a means to reduce standard errors of estimates rather than as the sole data from which the

88 area and accuracy estimates are produced.

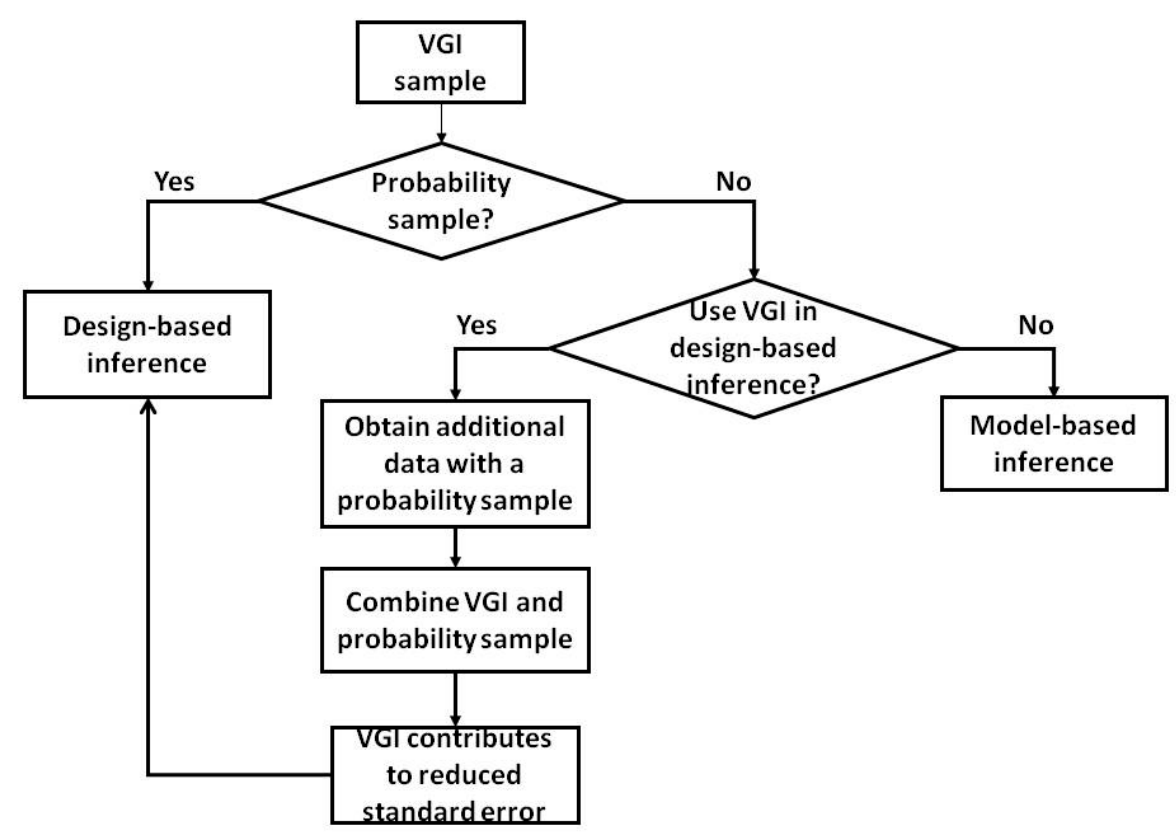

90 Figure 1. Schema for methodologies using VGI in accuracy assessment and area estimation. 
potential as a source of land cover information which is often constrained by lack of ground reference data. Second, the article provides methodological rigor and good practice advice for the use of data acquired via popular sample designs, ranging from judgmental to probability sampling. As such this article articulates methodology for producing credible inference from data sets that often do not conform to the requirements of widely used statistical inferential methods for two common and important application areas of remote sensing, accuracy assessment and area estimation. To do this, we, for the first time, synthesize methods developed in the general sampling literature into a comprehensive treatment of the theory and methods for using VGI in design-based inference. This includes translating methods developed for the use of non-probability samples for accuracy assessment and area estimation applications. As such we will show how VGI may be constructively used to decrease costs and reduce uncertainty (e.g., yield smaller standard errors and hence narrower confidence intervals) while following a methodology that allows for rigorous design-based inference. Throughout this article, guidance for using VGI in design-based inference is framed by examining the direct connection of the inference process to the three component protocols of accuracy assessment, the response design, sampling design, and analysis (Stehman and Czaplewski 1998).

The article is organized as follows. In Section 2, we define inference and describe the conditions

111 needed to satisfy design-based inference. Considerations regarding the use of VGI in design-based

112 inference are then explained in Section 3 in regard to the response design, sampling design and analysis

113 protocols. Section 4 provides the details of two methods for incorporating VGI in estimation of accuracy

114 and area that satisfy conditions of design-based inference, with both methods requiring that an

115 additional probability sample exists or could be acquired if the VGI did not originate from a probability

116 sampling design. Options for analysis when the only data available are VGI from a non-probability

117 sample are discussed in Section 5. Sections 6 and 7 provide discussion and a summary of the article. 
Following Baker et al. (2013, p.91), we define statistical inference as "... a set of procedures that

122 produces estimates about the characteristics of a target population and provides some measure of the

123 reliability of those estimates." Statistical inference focuses on the use of sample data to estimate

124 parameters of a target population, where a parameter is defined as a number describing the population

125 (e.g., the population mean and population proportion are two common parameters). Determining the

126 numerical value of a parameter would require a census of the study region, but in practice parameters

127 are estimated from a sample. Statistical inference also includes how bias and variance of these sample-

128 based estimators are defined. Baker et al. $(2013$, p.91) further specify that "A key feature of statistical

129 inference is that it requires some theoretical basis and explicit set of assumptions for making the

130 estimates and for judging the accuracy of those estimates." Consequently, sampling design and analysis

131 protocols must adhere to certain rules of implementation to ensure that the underlying mathematical

132 basis of the inference framework is satisfied. Failure to adhere to these rules may lead to substantial

133 bias in the estimators of parameters of interest or even nullify the ability to implement design-based

134 inference entirely (see Section 3.3).

Two general types of inference are design-based inference and model-based inference (De

136 Gruijter and Ter Braak 1990; Särndal et al. 1992; Gregoire 1998; Stehman 2000; McRoberts 2010, 2011).

137 In design-based inference, bias and variance of an estimator are determined by the randomization

138 distribution of the estimator which is represented by the set of all possible samples that could be

139 selected from the population using the chosen sampling design. This randomization distribution is

140 completely dependent on the sampling design hence the origin of the name "design-based" inference.

141 The inclusion probabilities of the sampling design are the critical link to the randomization distribution 
142

that underlies design-based inference (Särndal et al. 1992, section 2.4). The practical considerations for using VGI in design-based inference are explained in detail in Section 4.

A probability sampling design must satisfy two criteria related to the inclusion probabilities determined by the sample selection protocol. The inclusion probability of a particular element of the population (e.g., a pixel) is defined as the probability of that element being included in the sample. An inclusion probability is defined in the context of all possible samples that could be selected for a given sampling design. For example, if the design is simple random sampling of $n$ elements selected from the $N$ elements of the population, the inclusion probability of each element $u$ of the population is $\pi_{\mathrm{u}}=n / N$. That is, in the context of all possible simple random samples of size $n$ from this population, element $u$ has the probability of $n / N$ of being included in the sample selected. The two requirements of a probability sampling design are that $\pi_{\mathrm{u}}$ must be known for each element of the sample and $\pi_{\mathrm{u}}>0$ for each element of the population (Särndal et al. 1992; Stehman 2000). Probability sampling requires a randomization mechanism to be present in the selection protocol. Convenience, judgment, haphazard, and purposive selection of sample elements are examples of protocols that do not satisfy the criteria defining a probability sampling design (Cochran 1977, Sec. 1.6). Use of such samples for inference carries considerable risk due to lack of representation of the population.

An alternative to design-based inference is model-based inference (Valliant et al. 2000). As the name implies, model-based inference requires specification of a statistical model and inference is dependent on the validity of the model. Consequently, verifying model assumptions is a critical and often challenging feature of model-based inference. Model-based inference does not require a probability sampling design, although implementation of a probability sampling design is often recommended to ensure objectivity in sample selection because of the randomization (Valliant et al. 2000, p.20). Applications of model-based inference are briefly discussed in Section 5.3. 


\section{Component Protocols of Accuracy Assessment and Area Estimation}

We describe the role of each of the three components of the methodology (response design, sampling design, and analysis) in determining how VGI can be incorporated in rigorous design-based

170 inference. The response design is the protocol for determining the reference condition (i.e., the best 171 available assessment of the ground condition). The response design includes all steps leading to 172 assignment of the reference condition label of a point or spatial unit (e.g., a land-cover class or change 173 versus no change label). The sampling design is the protocol for selecting the sample units at which the 174 response design will be applied. Lastly, the analysis consists of defining parameters to describe 175 properties of the population (e.g., overall accuracy, proportion of area of each class) and the formulas 176 required to estimate these population parameters from the sample data. To justify the requirements of 177 each step to achieve the final accuracy or area estimates, our description starts with the analysis 178 (Section 3.1) focusing on how the VGI data would be used, followed by the steps of the response design 179 (Section 3.2) and the sampling design (Section 3.3).

\subsection{Analysis: Accuracy and Area Estimation Based on Totals}

The details of the analysis protocol that specify how the estimates of accuracy and area are produced yield insights into how VGI should be evaluated for use in design-based inference. The analysis focuses on summarizing information contained in an error matrix. We define the population to be a collection of $N$ equal-area units partitioning the region of interest. The population error matrix resulting from a census can be constructed in terms of area as illustrated by the numerical example in

187 Table 1 for a simple two-class legend, "crop" and "not crop" for a population (target region) of 1000 $\mathrm{km}^{2}$. The error matrix expressed in terms of area (Table 1) could easily be converted to proportion of 
matrix expressed in terms of area because we can formulate the population parameters of interest for

191 accuracy and area as totals or ratios of totals of areas. For example, overall accuracy is the total area of

192 agreement obtained from the sum of the area of the diagonal cells $\left(930 \mathrm{~km}^{2}\right)$ divided by the total area of

193 the target region $\left(1000 \mathrm{~km}^{2}\right)$ to yield overall accuracy of 0.93 or $93 \%$. User's accuracy for the crop class

194 is the total area where both the map and reference condition are crop $\left(840 \mathrm{~km}^{2}\right)$ divided by the total

195 area mapped as crop $\left(890 \mathrm{~km}^{2}\right)$ to yield the parameter 0.94 or $94 \%$. Producer's accuracy for the crop

196 class is the total area where both the map and reference condition are crop $\left(840 \mathrm{~km}^{2}\right)$ divided by the

197 total area of reference condition of crop $\left(860 \mathrm{~km}^{2}\right)$ to yield the parameter 0.98 or $98 \%$. Lastly, the area

198 of reference condition of the crop class is also simply a total, in this case the sum of the two cells in the

199 "crop" column of reference condition $\left(840+20=860 \mathrm{~km}^{2}\right)$.

200

201 Table 1. Population error matrix expressed in terms of area $\left(\mathrm{km}^{2}\right)$ for a hypothetical target region of

$2021000 \mathrm{~km}^{2}$. Overall accuracy is $93 \%(930 / 1000)$.

203

$\underline{\text { Reference Condition }}$

204

Map

Crop

Not Crop

Total User's

205

Crop

840

50

890

0.94

206

Not Crop

20

90

110

0.82

207 Total

860

140

1000

208 Producer's

0.98

0.64

Given that the parameters of interest for accuracy and area can be expressed in terms of totals,

211 the analysis focuses on estimating these totals. Basic sampling theory provides an unbiased estimator of

212 a population total in the form of the Horvitz-Thompson estimator (Horvitz and Thompson 1952). The

213 population total of the variable $y_{u}$ is defined as 


$$
Y=\sum_{P} y_{u}
$$

215 where the summation is over all $N$ elements of the population, $P$. For example, if $y_{u}$ is the area of crop

216 (as determined from the reference condition) for element $u$, then $Y$ is the total area of crop. The

217 population total $Y$ can be estimated from a sample using the Horvitz-Thompson estimator

$$
\hat{Y}=\sum_{s} \frac{y_{u}}{\pi_{u}}
$$

219 where the summation is over all elements of the sample $s$.

The Horvitz-Thompson estimator is an unbiased estimator of a population total for any sampling

221 design as long as the inclusion probabilities of the sample elements are known for that design. A useful

222 re-expression of the Horvitz-Thompson estimator highlighting the sample estimation weights is

$$
\hat{Y}=\sum_{s} w_{u} y_{u}
$$

224 where $w_{u}=1 / \pi_{u}$ is the estimation weight for element $u$ of the sample. Because $w_{u} \geq 1$, the $y_{u}$ value for 225 each sampled element is multiplied by an "expansion factor" $w_{u}$ to estimate a total. In effect each 226 sample element must account for itself along with some additional elements of the population that 227 were not selected into the sample. For example, for simple random sampling $w_{u}=N / n$ so $y_{u}$ for each 228 sampled element is "expanded" by the multiplier $w_{u}$ to account for $N / n$ elements of the population.

229 The critical importance of known inclusion probabilities for rigorous design-based inference is evident 230 via the role of the weights $w_{\mathrm{u}}=1 / \pi_{\mathrm{u}}$ in the estimator $\hat{Y}$ (equations 2 and 3 ).

Parameters such as user's accuracy and producer's accuracy are ratios of totals and

232 consequently can be estimated by the corresponding ratio of estimated totals (Särndal et al. 1992,

233 section 5.3). For example, if we define $Y$ as the total area of the population for which both the map and

234 reference condition are crop and $X$ as the total area mapped as crop, the ratio of population totals $Y / X$

235 would be the population parameter for user's accuracy of crop. User's accuracy could then be estimated

236 from the sample data using a ratio of Horvitz-Thompson estimators, $\hat{Y} / \hat{X}$, where both $\hat{Y}$ and $\hat{X}$ are

237 estimated totals based on equation (2), considering, respectively, $y_{u}=$ area of pixel $u$ with both map and 
reference condition of crop and $x_{u}=$ area of pixel $u$ mapped as crop. In the case of a pixel-based

239 assessment and assuming all pixels are equal area, user's accuracy of crop estimated using a ratio of

240 Horvitz-Thompson estimators would simply require defining $y_{u}=1$ if pixel $u$ has both map and reference

241 labels of crop ( $y_{u}=0$ otherwise) and defining $x_{u}=1$ if pixel $u$ has map label of crop ( $x_{u}=0$ otherwise). In

242 this formulation of user's accuracy, the ratio $Y / X$ is the proportion of pixels mapped as the target class

243 that have the reference label of that class.

Formulas for the variance and estimated variance of the Horvitz-Thompson estimator are

245 provided by Särndal et al. (1992, section 2.8). The square root of the estimated variance (standard

246 error) would be used to construct a confidence interval for the parameter of interest so issues of

247 inference obviously extend to variance and confidence interval estimation. Although we do not delve

248 into the details of the formulas for variance estimators, we emphasize that known inclusion probabilities

249 are an essential feature of variance estimation. Consequently, the requirement of implementing

250 probability sampling to ensure known inclusion probabilities for estimating a total applies as well to

251 estimating the variance of an accuracy or area estimator.

The conditions required for VGI to be used in design-based inference are apparent from the

253 analysis protocol. The accuracy and area parameters of interest can be expressed as population totals

254 or ratios of population totals and these totals can be estimated using the Horvitz-Thompson estimator.

255 From the Horvitz-Thompson estimator formula (equations 2 and 3) we observe that the key features of

256 VGI relevant to estimating a total are quality of the observation $y_{u}$ and knowledge of the inclusion

257 probability $\pi_{u}$. In other words, the questions pertinent to evaluating the utility of VGI for design-based

258 inference are: 1) What is the quality of $y_{u}$ (an issue to address in the response design) and 2) Is $\pi_{u}$

259 known (an issue to address in the sampling design)? The following two subsections address issues of

260 VGI related to the response and sampling designs. 


\subsection{Response Design}

The response design is the protocol for determining the reference condition of an element of the population. In the case of a land-cover legend based on a conventional hard classification, the response design results in a reference land-cover label assigned to each pixel (i.e., if the legend consists of $C$ classes, one and only one of these class labels is assigned to the pixel). The reference class labels can be translated to a quantity by the simple process of defining $y_{u}=1$ if pixel $u$ has reference class $c$ and $y_{u}=0$ otherwise. Thus for example if class $c$ is forest, all pixels with reference class forest would be assigned $y_{u}=1$ and all non-forest pixels would have $y_{u}=0$. Evaluating and assuring the quality of VGI is critical because high quality reference data are absolutely essential to accuracy and area estimation. If the reference labels are not accurate, these errors can have a substantial impact on accuracy and area estimates (Foody 2009, 2010). Very accurate reference data obtained within a timeframe corresponding to the date of remote sensing image acquisition are a necessity for every application of accuracy assessment and area estimation from remote sensing. VGI has considerable potential as a source of reference data, notably in facilitating the collection of a large set of observations over broad geographical regions. However, the use of volunteers rather than experts in assigning the reference class labels may exacerbate concerns regarding label accuracy, although amateurs can sometimes be as accurate as experts in labeling (See et al. 2013). Further, VGI tends to be collected continuously rather than within a narrow time frame which can limit its value, especially for studies of land-cover change.

Applications in which VGI has been collected for land cover and land use studies are becoming increasingly common. Fonte et al. (2015) reviewed several applications including: 1) Geo-Wiki project, which uses the crowd for interpretation of very high resolution satellite imagery (Fritz et al. 2012);

2) VIEW-IT, which is a validation system for MODIS land cover (Clark and Aide 2011); and 
3) geo-tagged photographs for land cover validation from different applications such as the

Degree Confluence Project, Geograph, Panoramio and Flickr (Antoniou et al. 2016; Fonte et al. 2015; Iwao et al. 2006).

Another source of VGI for land-cover studies is the LACO-Wiki system, an online land cover validation

tool intended as a repository of openly available validation data crowdsourced from different users (See et al. 2017). More recently, land cover and land use have been crowdsourced in the field through the FotoQuest Austria app, which sends users to specific locations and loosely follows the LUCAS protocol

292 for data collection (Laso Bayas et al. 2017). Hou et al. (2015) describe geo-tagged web texts as an 293 alternative to photographs as yet another source of VGI useful for land-cover studies.

The quality of the VGI data collected for land cover and land use studies has received recent 295 attention. A substantial body of literature focuses on the positional quality and completeness of 296 OpenStreetMap (OSM), the most commonly cited VGI project (e.g., Ciepłuch et al. 2010; Girres and 297 Touya 2010; Haklay 2010). Other elements of quality include thematic accuracy (which is relevant to 298 land cover and land use), temporal quality, logical consistency, and usability, all of which are set out in 299 ISO 19157 (Fonte et al. 2017a). In addition, Antoniou and Skopeliti (2015) outline quality indicators that 300 are tailored to VGI such as data indicators, demographic and other socio-economic indicators, and 301 indicators about the volunteers. Due to the specificities of VGI when compared to traditional 302 geographic information and the diversity of uses of these data, additional methodologies are starting to 303 be developed that aim to integrate several quality measures and indicators into quality assessment 304 workflows, enabling quality data to be combined to produce more reliable quality information (e.g., 305 Bishr and Mantelas 2008; Jokar Arsanjani and Bakillah 2015; Meek et al. 2016).

307 methods such as latent class analysis, which can be used to characterize volunteers in terms of their 308 quality in labeling classes and could therefore be used to filter or weight the data when used 
subsequently in applications (Foody et al. 2013, 2015). These issues of data quality associated with the

310 response design are critical to the overall process of accuracy and area estimation. In reality, reference

311 data quality issues are equally impactful whether the source of the reference classification is VGI or

312 expert interpretation (See et al. 2013).

313

$314 \quad 3.3$ Sampling Design

315 The sampling design is the protocol used to select the subset of locations (e.g., pixels) at which

316 the reference condition is determined. As noted earlier, the inclusion probability of pixel $u$ is denoted as

$317 \pi_{u}$, and the two criteria defining a probability sampling design are: 1) $\pi_{u}$ is known for all pixels in the

318 sample and 2) $\pi_{u}>0$ for all pixels in the population. Because probability sampling is a requirement of

319 rigorous design-based inference, the sample selection protocol must ensure that these two conditions

320 of $\pi_{\mathrm{u}}$ are satisfied. Moreover, randomization of the sample selection is required of all probability

321 sampling designs as it is this randomization that creates the probabilistic foundation for design-based

322 inference. The sampling design is linked to the analysis via the inclusion probabilities that are

323 incorporated in the Horvitz-Thompson estimator (equations 2 and 3).

Because design-based inference requires known inclusion probabilities, it is critical to establish

325 whether a probability sampling design was the basis for collecting VGI data. The distinction between

326 active and passive VGI is relevant in this regard. Active VGI refers to directing volunteers to specific

327 sample locations (e.g., See et al. 2016) and therefore allows for implementing a probability sampling

328 design for collecting VGI. Conversely, passive VGI refers to allowing volunteers to choose where they

329 will collect data and typically leads to purposive or convenience sampling with attendant concern

330 regarding lack of representation of the full population. The protocols that determine where VGI data

331 are collected span a continuum ranging from rigorous probability sampling to selection by judgment or

332 convenience without an underlying random mechanism. 
334 via a probability sampling protocol. These data are obtained at locations defined by the intersection of 335 lines of latitude and longitude and therefore originate from a design akin to systematic sampling (due to 336 the Earth's shape the distances between sample points vary with latitude so the inclusion probabilities

337 would not all be equal but would still be known). A second example of VGI based on a probability 338 sampling design is the FotoQuest Austria app which uses the Land Use/Cover Area frame Survey (LUCAS) 339 sample (which is based on a systematic sample of points spaced $2 \mathrm{~km}$ apart in the four cardinal 340 directions across the European Union) followed by a stratified sample (Martino et al. 2009). That is, land 341 cover and land use were crowdsourced via the FotoQuest Go mobile app in which volunteers were sent 342 to specific locations that formed part of the LUCAS systematic sample for Austria, and the LUCAS sample 343 was then augmented with additional sample units (Laso Bayas et al. 2016). and volunteer chosen locations. The Geo-Wiki project is used to collect land cover and land use data via different campaigns (See et al. 2015). These campaigns have all had different purposes and hence were

347 driven by different sampling designs. For example, the first campaign to validate a map of land 348 availability for biofuels was driven by a stratified random sample with equal sample size in both the land available stratum and the land unavailable stratum. To this an additional sample from cropland areas

350 was added although the data were not used to undertake an accuracy assessment as such but to modify 351 the statistics on how much land is available (Fritz et al. 2013). Other studies have made use of Geo-Wiki 352 data from previous campaigns for validation that were not obtained using a probability sampling 353 approach for the specific product to be validated (see, for example, Schepaschenko et al. (2015) and 354 Tsendbazar et al. (2015) for review of reference datasets including those from Geo-Wiki). The VIEW-IT 355 application (Clarke and Aide 2011) either directs users to specific locations selected based on a 356 probability sampling design or users can provide information about the land cover at any location, which 
means these latter sample locations would not be part of a probability sampling design. The LACO-Wiki

358 system (See et al. 2017) has built-in probability sampling schemes although users can upload their own sample locations that do not necessarily conform to a probability sampling design.

Photograph repositories such as Panoramio, Flickr, and Instagram are examples of passive VGI

361 and therefore do not conform to any probability sampling design. For example, photographs made

362 available by citizens may be positioned at any location chosen by the volunteer (such as the

363 photographs available in Flickr or Instagram), or collected at predefined locations. Similarly, the data

364 available in collaborative projects such as OSM are created at locations of interest to the citizen

365 volunteers, and consequently these data have no underlying probability sampling design. The amount

366 and quality of the OSM data are known to be correlated with demographic or socio-economic factors

367 (e.g., Mullen et al. 2014; Elwood et al. 2013) and this offers some possibility for adjusting estimates to

368 account for misrepresentation of the population (see Section 5.1).

The Geograph project asks users to take photographs in every square kilometer of the United

370 Kingdom and classify them (now also extended to other locations in the world). Since $2005,83.4 \%$ of

371 the $1 \mathrm{~km}^{2}$ squares in Great Britain and Ireland have photographs (http://www.geograph.org.uk/,

372 accessed 29 October 2017) and nearly 5.5 million images are available within this time period.

373 Volunteers may choose locations within each square kilometer at which photographs are taken.

374 Therefore, if each photograph is viewed as representing a point location or, for example, the $30 \mathrm{~m} \times 30$

375 m pixel surrounding the photograph's location, the data would not meet the criteria defining a

376 probability sampling design due to the lack of randomization in the selection protocol. Directing the

377 volunteers to cover the $1 \mathrm{~km}^{2}$ squares provides a better degree of spatial representation of the VGI than

378 might otherwise occur if volunteers are allowed to choose locations completely on their own.

379 Specifically, the $1 \mathrm{~km}^{2}$ squares effectively serve as spatial (geographic) strata, and with over $83 \%$ of

380 these strata visited, the Geograph project data achieve the desirable design criterion of being spatially 
381 well distributed (Stehman 1999, Figure 3). The Geograph project data collection protocol illustrates the

382 fact that within the class of non-probability sample designs, features can be built into the protocol to 383 enhance representation of the VGI data.

384

\section{4. Methods to Use VGI in Design-based Inference}

In this section, we address how to incorporate VGI into design-based inference focusing on

387 sampling design and estimation considerations (Figure 2). The label quality issues of VGI remain a

388 concern but are not addressed in this section. The most straightforward approach to ensure the utility

389 of VGI for design-based inference is to direct volunteers to collect data at locations specified by a

390 probability sampling design (which is possible with "active VGI"). Several examples of VGI collections

391 based on a probability sampling design were documented in Section 3.3. Specifying sample locations

392 selected via probability sampling has the potential drawback that volunteer participation may be

393 reduced if volunteers are unable to choose locations of personal interest. Consequently, additional

394 effort may be necessary to obtain $y_{u}$ at those locations neglected by volunteers. 


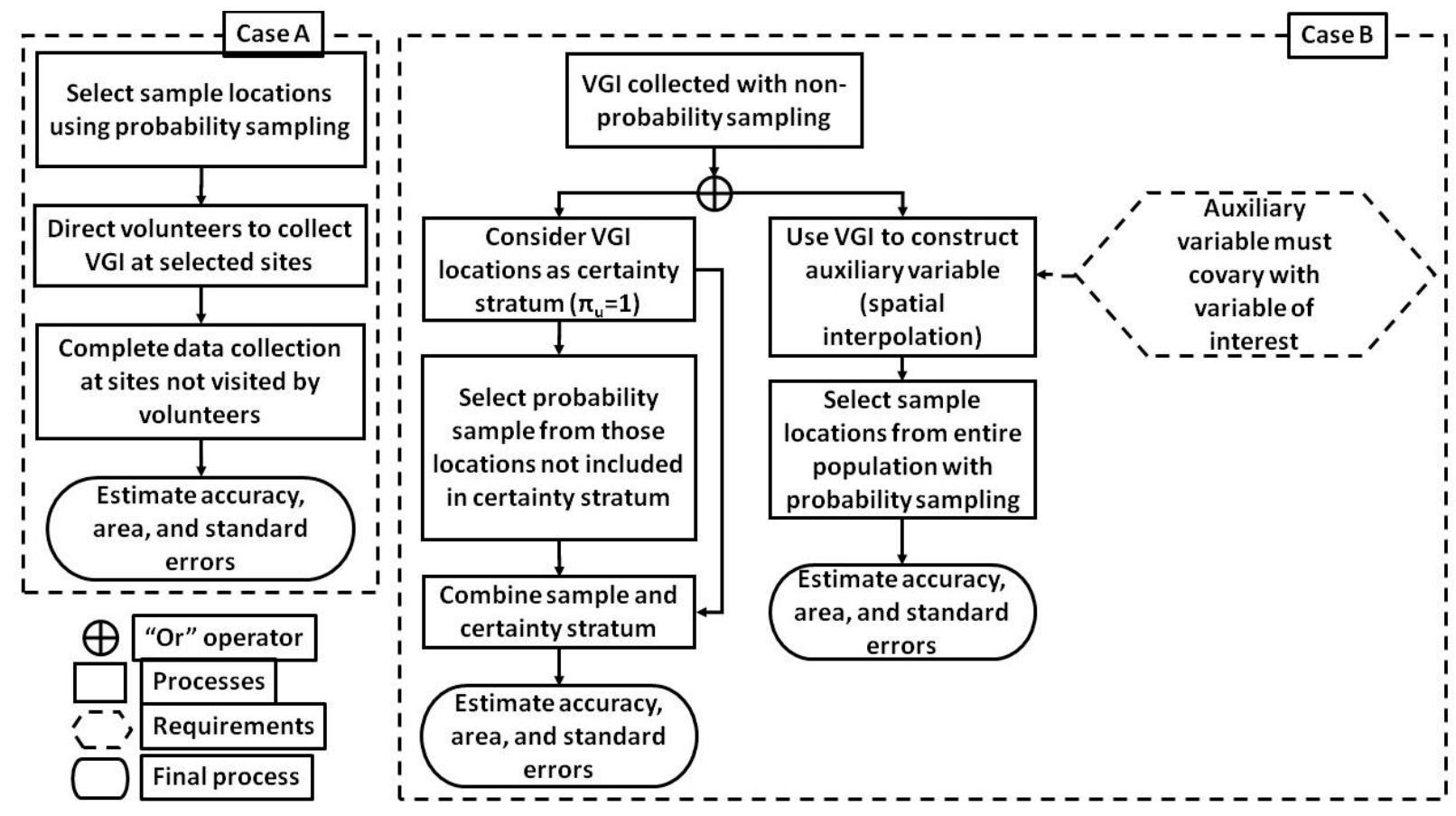

396

Figure 2. Schema for using VGI in design-based inference.

If a large quantity of VGI obtained from a non-probability sampling design exists, the VGI data may be augmented with data from a probability sampling design (Figure 2). Two options are described in the following subsections. In the first option, the VGI data are treated as a "certainty stratum" and combined with data from a probability sample selected from the locations not already included in the VGI data. In the second option, the probability sample is selected from the full population and the VGI data are used to construct an auxiliary variable that is then incorporated in a model-assisted estimator to reduce the standard errors of the estimates based on the data from the probability sample.

\subsection{VGI Incorporated as a Certainty Stratum}

VGI data can be combined with data obtained from a probability sample by treating each VGI sample unit (e.g., a pixel) as belonging to a "certainty stratum" in which the inclusion probability is $\pi_{u}=1$ (Overton et al. 1993). By assigning $\pi_{\mathrm{u}}=1$ to each VGI sample unit, we acknowledge that these sample units were not selected via a randomized selection protocol, and instead we view these units as having 
411 been purposely selected to be included with certainty in the sample. From the remaining units of the

412 population not included in the VGI certainty stratum, a probability sampling design is implemented and

413 these newly selected sample units are combined with the VGI data to produce the accuracy and area

414 estimates. In this approach the VGI data are used directly in the estimation of accuracy and area, so the 415 quality of the VGI data is a critical concern.

All sample units selected via the probability sampling design will have a known inclusion

417 probability and the data from these sample units can be combined with the VGI data using the Horvitz-

418 Thompson estimator. Specifically, suppose there are $N_{1}$ elements for which we have no VGI and $N_{2}$

419 elements for which VGI provides $y_{u}\left(N=N_{1}+N_{2}\right)$. Further, let $G$ denote the subset for which VGI is

420 available (the "G" is from the middle letter of VGI) and $\tilde{G}$ denote the subset of the population for which

$421 \mathrm{VGI}$ is not available. The population total $Y$ can then be partitioned into summations over the two

422 subpopulations $\widetilde{G}$ and $G$,

423

$$
Y=\sum_{\tilde{G}} y_{u}+\sum_{G} y_{u}=Y_{\tilde{G}}+Y_{G}
$$

424 Because $Y_{G}$ (total of $y_{u}$ for the VGI data) is known, it is only necessary to estimate $Y_{\tilde{G}}$ from the sample.

425 Therefore, an estimator of $Y$ can be expressed as

426

$$
\widehat{Y}=\sum_{s} y_{u} / \pi_{u}+\sum_{G} y_{u}=\widehat{Y}_{\tilde{G}}+Y_{G}
$$

427 where the first summation is over the elements selected in the sample from the $N_{1}$ elements of the 428 population $\tilde{G}$ for which VGI is not available. The variance of $\hat{Y}$ is $V(\widehat{Y})=V\left(\widehat{Y}_{\tilde{G}}\right)$ because the total of the 429 VGI data is a known quantity with no uncertainty attributable to sampling. That is, the only uncertainty 430 attributable to sampling arises from estimating the total $Y_{\tilde{G}}$ for the non-VGI portion of the population, $431 \tilde{G}$.

The benefit of the VGI data when incorporated as a certainty stratum is to reduce the standard 433 errors of the accuracy and area estimators and accordingly to decrease the width of confidence intervals 434 for the parameters of interest. To illustrate the potential reduction in standard error, we focus on the 
objective of estimating area based on the reference condition obtained for each sample unit. The

436 benefit of the VGI data can then be quantified by comparing the variance of the estimator of total area

437 without using VGI data to the variance of the estimator using the certainty stratum approach (equation

438 5). Several conditions are imposed to simplify the variance comparison: 1) the sample of non-VGI units

439 is selected by simple random sampling; 2 ) the VGI data have the same variability as the non-VGI data

440 (i.e., the variance of $y_{u}$ for the VGI subpopulation $G$ is the same as the variance of $y_{u}$ for the non-VGI

441 subpopulation $\tilde{G}$ ); and 3) the sample size $n$ is the same regardless of whether VGI is present (i.e., the VGI

442 data are viewed as obtained at no cost so $n$ is the same with or without VGI). If no VGI data are

443 available and a simple random sample is selected from the full population of $N$ elements (i.e., $N_{2}=0$

444 because no VGI data exist), the variance of the estimated total is

445

$$
V(\hat{Y})=N^{2}\left(1-\frac{n}{N}\right) V_{y} / n
$$

446 The variance of $\hat{Y}$ when VGI is available for $N_{2}$ elements of the subpopulation $G$ is derived as follows. A

447 simple random sample of $n$ elements is selected from the $N_{1}$ non-VGI units. The variance of the

448 estimated total combining the VGI data with the non-VGI sample (equation 5 ) depends only on the

449 variance of the total estimated from the non-VGI sample units,

$$
V\left(\widehat{Y}_{\tilde{G}}\right)=N_{1}^{2}\left(1-\frac{n}{N_{1}}\right) V_{y} / n
$$

451 To quantify the reduction in variance achieved by the VGI data, we examine the ratio of the two

452 variances,

$$
R=\frac{V\left(\hat{Y}_{\widetilde{G}}\right)}{V(\hat{Y})}=\frac{N_{1}^{2}\left(1-\frac{n}{N_{1}}\right)}{N^{2}\left(1-\frac{n}{N}\right)}
$$

454 The $V_{y} / n$ term common to both equations (6) and (7) cancels in the ratio $R$ by virtue of the assumption 455 that the variability of $y_{u}$ is the same in the VGI and non-VGI subpopulations (if $V_{y}$ is different in the two 456 subpopulations, $R$ will be impacted by the ratio of the variances of the two subpopulations, $G$ and $\tilde{G}$ ). 
458 reduce variance depends on the proportion of the population that is covered by the VGI data, which is 459 defined as $k=N_{2} / N$. If we define $f=n / N$ to be the proportion of the total population selected for the 460 probability sample, then $R$ can be re-written as

$$
R=(1-k)(1-f-k) /(1-f) .
$$

462 If no VGI data exist, then $k=0$ and $R=1$ as expected because there would be no reduction in variance

463 from VGI. Conversely, if $k=1$, then $R=0$ as expected because the VGI would constitute a census and the 464 population total $Y$ would be known yielding a variance of 0 . As the quantity of VGI gets larger (i.e., $465 k=N_{2} / N$ increases), $R$ decreases indicating a greater benefit accruing to the availability of the VGI data.

466 Numerical values of $\sqrt{R}$ (ratio of standard errors) for several combinations of $k$ and $f$ are presented in 467 Table 2. For a fixed value of $f=n / N, \sqrt{R}$ decreases approximately linearly with increasing $k$. For a fixed 468 value of $k$, the decrease in $\sqrt{R}$ is much less prominent as $f$ increases except for the case with $f=0.25$ and $469 k=0.75$ which represents a census so $V\left(\hat{Y}_{\tilde{G}}\right)=0$. To simplify the problem still further, assume that the 470 spatial unit of the assessment is a pixel and that $N$ is so large that $f=n / N=0$. Then setting $f=0$ in 471 equation (9), we obtain $R=(1-k)^{2}$ which leads directly to

$$
\sqrt{R}=1-k
$$

473 Thus for very large populations the reduction in standard error achieved by VGI will be directly related

474 to $k$, the proportion of the population for which VGI is available - the greater the quantity of VGI 475 available (i.e., larger $k$ ) the greater the reduction in standard error. 
Table 2. Reduction in standard error achieved by using VGI in the certainty stratum approach. Values

481 shown in the table are $\sqrt{R}$ where $\mathrm{R}$ is the ratio of the variance of the estimated total with VGI data

482 incorporated in a certainty stratum divided by the variance of the estimated total in the absence of VGI

483 (see equations 8 and 9). Ratios are provided for different combinations of $k=N_{2} / N$ (the proportion of

484 the region of interest covered by VGI) and $f=n / N$ (proportion of the study region covered by the simple 485 random sample).

486

487

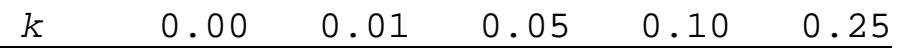

488

0.01

$\odot .99$

0.99

0.99

0.99

0.99

489

0.05

0.95

0.95

0.95

0.95

0.94

490

0.10

0.90

0.90

0.90

0.89

0.88

491

0.25

0.75

0.75

0.74

0.74

0.71

492

0.50

0.50

0.50

0.49

0.47

0.41

493

0.75

0.25

0.25

0.23

0.20

0.00

494

0.90

0.10

0.10

0.07

0.00

0.00

495

Equation (9) and the results of Table 2 can be used to examine the benefit of VGI arising from

497 photographs contributed by volunteers (Antoniou et al. 2016), a common source of VGI for land-cover

498 studies. Suppose we assume a photograph to be representative of a $30 \mathrm{~m} \times 30 \mathrm{~m}$ pixel and consider a

499 region of interest that covers 8 million $\mathrm{km}^{2}$ (roughly the size of the conterminous United States,

500 excluding Alaska and Hawaii). This region would have approximately $N=9$ billion pixels. To achieve a

$5015 \%$ reduction in the standard error of the estimated area of a targeted class (i.e., $\sqrt{R}$ changes from 1 to

5020.95 ) the certainty stratum approach would require $k=N_{2} / N=0.05$ which translates to needing $N_{2}=450$

503 million photographs. As a second example, suppose the target region of interest covers $100,000 \mathrm{~km}^{2}$

504 (area slightly larger than Portugal). This population would have $N=100$ million pixels $(30 \mathrm{~m} \times 30 \mathrm{~m})$ so 
for VGI data to contribute a $5 \%$ reduction in standard error we would need $N_{2}=5$ million photographs.

506 Typically the VGI photographs will have to be processed to obtain the land-cover information of interest

507 (e.g., a land-cover class). Consequently, the large number of photographs needed in these examples to

508 achieve only a 5\% reduction in standard error would require substantial computer processing capability

509 and possibly automated methods to identify the land-cover class from the photographs. Accordingly,

510 the response design effort to process such large numbers of photographs may make this use of VGI cost

511 prohibitive in some applications.

512 The certainty stratum approach may have greater utility when the VGI data are in the form of

513 fully mapped areas classified to a land-cover or change type (i.e., in contrast to individual, unlabeled

514 photographs as in the previous paragraph). For example, Fonte et al. (2017b) described an application

515 in which OSM provided land-cover information for two study areas of $100 \mathrm{~km}^{2}$ in London and Paris.

516 OSM coverage was $88 \%$ for the London region and $97 \%$ for the Paris region. Because of the substantial

517 portion of area covered by OSM ( $k=0.88$ for London and $k=0.97$ for Paris) a large reduction in standard

518 error of accuracy and area estimates would be expected by using these OSM data in the certainty

519 stratum approach. For example, if $k=0.88$ and $\mathrm{f}=0.1$ (the London example), we obtain $R=0.00266$

$520(\sqrt{R}=0.05)$ indicating that the standard error of the certainty stratum estimator would be $5 \%$ of the

521 standard error of the estimated area when not using the VGI from OSM. Obviously the areas of the

522 regions of interest for the OSM examples in this paragraph are much smaller than for the examples in

523 the previous paragraph and $k$ would surely be smaller if OSM were to be used for national estimates.

$525 \quad 4.2$ Use of VGI in a Model-Assisted Estimator

Brus and de Gruijter (2003) developed an approach to use data from a non-probability sampling

527 design to produce estimates within the design-based inference framework. In this approach, a spatial

528 interpolation method is applied to the non-probability sample of VGI data to construct an auxiliary 
variable for all $N$ elements of the population. The auxiliary variable is then used in a model-assisted

530 estimator to achieve a reduction in standard error. Model-assisted estimators represent a broad class of estimators in which one or more auxiliary variables are incorporated in the estimator. Common examples of model-assisted estimators include difference, ratio, and regression estimators as well as post-stratified estimators (Särndal et al. 1992; Gallego 2004; Stehman 2009; McRoberts 2011; Sannier et al. 2014). The auxiliary variables are expected to covary with the target variable of interest and the information in the auxiliary variables, when incorporated in the model-assisted estimator, thus serves to reduce standard errors (Särndal et al. 1992, Chapter 6).

The Brus and de Gruijter (2003) approach could be applied to VGI as follows. Consider the objective of estimating the proportion of area of a class (e.g., area of forest) based on the reference condition. Suppose the spatial unit of the analysis is a pixel and the VGI data consist of $N_{2}$ pixels labeled 540 as forest or non-forest. The Brus and de Gruijter (2003) approach uses these VGI data to construct an 541 auxiliary variable $x_{u}$ for all $N$ pixels in the population. For example, for a binary classification of forest / 542 non-forest, the auxiliary variable would be defined as $x_{u}=1$ if the class is forest and $x_{u}=0$ if the class is 543 non-forest. The auxiliary variable $x_{u}$ is known for the $N_{2}$ pixels comprising the VGI, and the Brus and de 544 Gruijter (2003) approach would then implement a spatial interpolation method such as indicator kriging 545 (e.g., Isaaks and Srivastava 1989) to predict values of $x_{u}$ for the $N-N_{2}$ pixels not included in the VGI 546 subset of the population. The binary forest / non-forest classification of the region predicted from the 547 VGI data could be used in the same manner as auxiliary data from any forest / non-forest map. For 548 example, to estimate the proportion of area of forest based on the reference condition $\left(y_{u}\right), a$ 549 probability sample from all $N$ pixels would be selected for which the reference class of each sampled 550 pixel would be obtained. If the reference observation is also a binary forest / non-forest classification 551 (i.e., $y_{u}=1$ if the reference condition is forest, $y_{u}=0$ otherwise), an error matrix could be estimated from 552 the sample based on the reference class data and the map classification of forest or non-forest created 
from the VGI data. The error matrix information could then be combined with the VGI generated forest /

554 non-forest map information to produce a post-stratified estimator of the proportion of area (Card 1982;

555 Stehman 2013). The expectation is that the auxiliary variable created from the VGI would yield a

556 reduction in standard error of the post-stratified estimator relative to an estimator that did not

557 incorporate the VGI. That is, the map generated via spatial interpolation of the VGI data would be used

558 in the same way that a forest / non-forest map derived from remotely sensed data would be used in a

559 post-stratified estimator.

The Brus and de Gruijter (2003) method requires a probability sample to provide the reference

561 data $\left(y_{\mathrm{u}}\right)$ for the accuracy and area estimates. This probability sample must be selected from the full

562 population of $N$ units, including those units for which VGI is available. In contrast, the certainty stratum

563 use of VGI (section 4.1) does not require a sample from the subpopulation $G$ that has VGI. The Brus and

564 de Gruijter (2003) approach does not use the VGI data as the observed response (i.e., the reference data

565 value, $y_{u}$ ) so the quality of the class labels associated with the VGI data will not impact the estimates in

566 terms of potential bias attributable to labeling error of the VGI. However, better quality (i.e., more

567 accurate) VGI data would likely yield a greater reduction in standard error in the same manner that a

568 more accurate map yields a greater reduction in standard error when the map data are used in a post-

569 stratified estimator (Stehman 2013). In the context of land-cover accuracy and area estimation

570 applications, remote sensing information is almost always available to produce a map that would

571 provide auxiliary information that could be used in a model-assisted estimator. Spatial interpolation of

572 VGI using the methods described by Brus and de Gruijter (2003) provides another option for producing a

573 map of auxiliary information, and incorporating remote sensing imagery in linear spatial models (Diggle

574 et al. 1998) might further enhance the precision benefit of the Brus and de Gruijter (2003) approach. 
577 the concern with bias attributable to inaccurately labeled VGI data. Instead, the approach employs the

578 VGI to create an auxiliary variable $x_{u}$ that is then used in a model-assisted estimator to reduce the

579 standard errors of the accuracy and area estimates. The magnitude of the reduction in standard error

580 would depend on the quality of the VGI. While this approach would have great utility if no other

581 auxiliary information were available, we typically have access to remotely sensed data that could be

582 used to produce a classification that would serve the same purpose as a map derived from spatially

583 interpolating VGI data. Consequently, for land-cover studies the primary benefit obtained by spatial

584 interpolation of VGI may occur in circumstances where a map produced from remotely sensed data is

585 not available.

586

\section{5. Use of VGI from Non-Probability Samples}

If the VGI data are the only source of reference data (i.e., there is no probability sample and

unable to acquire one), it will be challenging to use these VGI data in the manner of design-based

590 inference (Figure 3). One option for using VGI in this context is to replace the estimation weights

$591 w_{u}=1 / \pi_{\mathrm{u}}$ (equation 3 ) by pseudo weights that depend on assuming the sample can be treated as though

592 it had been obtained via a probability sampling design. For example, suppose the reference data for

593 accuracy assessment and area estimation are land-cover interpretations extracted from a non-

594 probability sample of photographs. If the inclusion probabilities $\left(\pi_{u}\right)$ of the spatial units represented by

595 these photographs are unknown, one approach to estimate totals is to assume that the VGI locations

596 represent a stratified random sample (see Section 5.1 for details). Using this approach it is possible to

597 construct pseudo-weights such that estimated totals will match known parameters of the population.

598 Although this weighted estimation approach can adjust a VGI sample to achieve estimates that

599 correspond to the correct proportional representation of the population, the question of "external

600 validity" of the VGI data must be addressed. External validity is defined and applied in Section 5.2. 


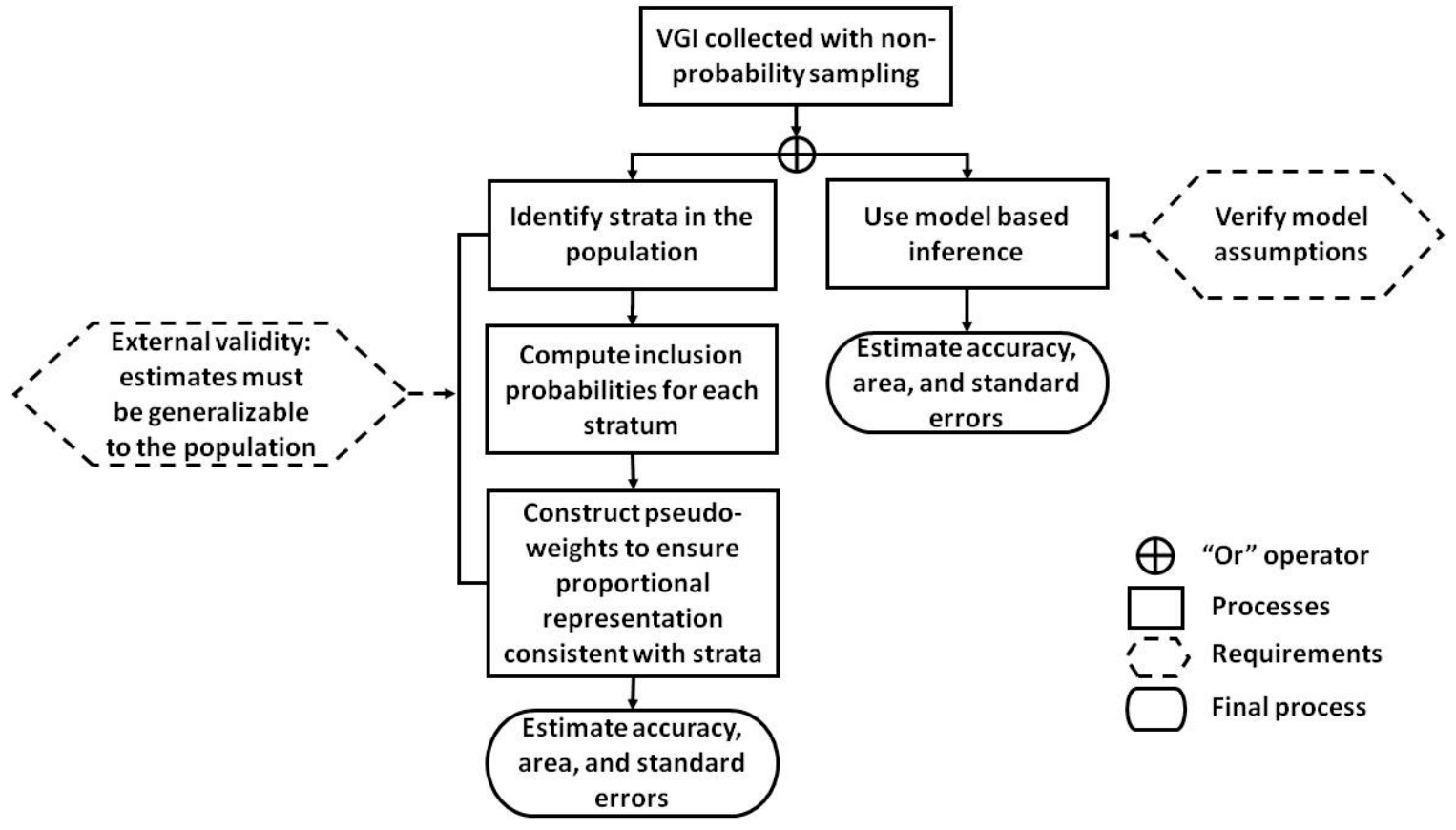

Figure 3. Schema for using VGI collected via a non-probability sampling design.

\subsection{Estimation Based on Pseudo-Weights}

If the only reference data available for accuracy and area estimation are VGI that did not originate

611 land-cover map of the study region, we could compare the proportion of the VGI data found within each

612 land-cover class to the proportion of each class in the entire population. For the hypothetical numerical

613 example of Table 3, the VGI sample shows preferential selection from the developed and crop classes at

614 the expense of representation of the "other" and natural vegetation classes reflecting the relative ease

615 of access to the classes associated with the transport network. Representativeness of the VGI data 
could also be assessed by examining the distribution of distances to the nearest road or distances to the

617 nearest population center. For example, we could compare the mean distance to the nearest road for

618 the VGI locations to the mean distance for all $N$ pixels in the population. If the mean for the VGI

619 locations was less than the mean for the population, this discrepancy would indicate preferential

620 selection of VGI closer to a road. A relevant question is then whether this preferential selection could

621 introduce bias because map accuracy may differ depending on proximity to a road.

622

623 Table 3. Hypothetical data illustrating evaluation of the proportional representation of VGI. The

624 distribution of the percent area of the map classes is compared between the VGI sample $(n=100)$ and

625 the population (i.e., entire region) known from a land-cover map of the study region.

626

627

Area (\%)

628

Map Class

VGI Population

629

Developed

25

10

630

Crop

35

20

631

Natural vegetation

30

50

632 Other

10

20

633

In general, we could attempt to adjust estimates to account for recognized non-proportionality of the VGI data relative to known population characteristics (Dever et al. 2008). For the example data of

636 Table 3, the difference between the distribution of the VGI and population data suggests that weighting

637 the data to adjust for this discrepancy would be a good idea when producing estimates. One approach

638 would be to construct weights such that the estimates based on the weighted analysis of the VGI data

639 correspond to known population quantities. A simple way to achieve this is to treat the non-probability 
641 for each stratum are then defined as $\pi_{u}=n_{h} / N_{h}$ where $n_{h}$ is the observed sample size (from the VGI

642 sample) in stratum $h$ and $N_{h}$ is the population size in stratum $h$. The estimation weight for pixel $u$ is then

$643 w_{u}=1 / \pi_{u}$, and these weights could be used in the Horvitz-Thompson estimator. These stratified

644 estimation pseudo-weights for the hypothetical data of Table 3 are presented in Table 4. Referring to 645 weights constructed in this manner as "pseudo-weights" highlights the fact that they are not derived 646 from inclusion probabilities generated by a probability sampling protocol.

647

648 Table 4. Pseudo-weights for VGI sample units based on distributions by class shown in Table 3 ( $n_{h}$ and $649 N_{h}$ represent the number of pixels for each class in the VGI sample and in the population).

650

651

$$
n_{h} \quad N_{h}
$$

652 Class VGI Map $w_{u}=\underline{N}_{h} \underline{L} \underline{n}_{h}$

$653 \quad$ Developed $\quad 25 \quad 1000 \quad 40$

$654 \quad$ Cultivated $\quad 35 \quad 2000 \quad 57$

$655 \quad$ Natural veg $\quad 30 \quad 5000 \quad 167$

656 Other $\quad 10 \quad 2000 \quad 200$

657 Total $\quad 100 \quad 10000$

658

659 To illustrate how the stratified estimation approach using pseudo-weights is implemented, consider 660 estimating the proportion of area mapped as the developed class. From Table 3, we know this 661 proportion is 0.10 because we have the map for the entire population. How well does the VGI sample 662 estimate this parameter? We observe that 25 out of 100 VGI pixels are mapped as developed so the 663 estimated proportion of mapped developed is then 0.25 from the VGI data, greater than the known 
parameter of 0.10 for the population. To produce the estimator using the stratified pseudo-weights of

665 Table 4 we define $y_{u}=1$ if the sample pixel has the map label of developed and $y_{u}=0$ otherwise. Then for 666 the developed class stratum, $y_{u}=1$ for all 25 sample pixels and each of these pixels has a weight of

$667 w_{u}=40$, so the estimated total contributed from this stratum is $40 \times 25=1,000$ pixels (using equation 3 ).

668 For the other three strata, $y_{u}=0$ for all sample pixels so these strata contribute no additional pixels to the 669 estimated number of mapped developed pixels. Dividing the estimated total number of map pixels 670 labeled as developed $(1,000)$ by the number of pixels in the population $(N=10,000)$ yields an estimated 671 proportion of 0.10 which matches the population proportion of mapped developed area from Table 3.

672 Thus the sample estimate using the pseudo-weights matches this known population proportion.

673 In general, the pseudo-weights can be constructed so that the sample estimates will equal known 674 population values. In the example of Table 4, the pseudo-weights reproduce the known values $675 N_{h}=$ population size of each stratum, a property known as "proportional representation." These same 676 estimation pseudo-weights are then applied to estimate the target population parameters and the 677 assumption is that estimation weights that effectively adjust the VGI sample data to match known 678 population parameters will also work well when estimating the target parameters for which we do not 679 have full population information. Other more complex methods for creating estimation weights include 680 raking, general calibration estimators (Deville and Särndal 1992), and propensity scores (Valliant and 681 Dever 2011). Models can be used to produce the pseudo-weights used in lieu of weights that are the 682 inverse of the inclusion probabilities of a probability sampling design, but Valliant $(2013$, p.108) points 683 out that this approach has not yielded promising results because the models are weak and the 684 requirements excessive for covariates to be used in the models. 685 
Pseudo-estimation weights can be used to produce estimates that capture the proportional distribution of known population characteristics (i.e., covariates). However, another important aspect of representativeness of non-probability sample data is external validity, defined as the parameter estimates being "generalizable outside the sample, say to the population of interest" (Dever and Valliant 691 2014). For the pseudo-weight estimation approach described in the previous section, establishing external validity would require that accuracy for the subset of the population represented by the VGI locations be equivalent to accuracy of the full region. Proportional representation of the estimates

694 (Table 4) produced from non-probability sample data is one aspect of external validity, but proportional 695 representation is not sufficient to establish external validity (Dever and Valliant 2014). same as the population of the full study region. Two examples are provided to illustrate this practical 698 issue. In both examples, the objective is to estimate the accuracy of a map. For the first example, 699 suppose that volunteers avoid locations of complex land cover and provide reference data exclusively for locations that are surrounded by homogeneous land cover. Antoniou et al. (2016) suggest such a 701 strategy may be beneficial when using photographs to avoid difficulties of determining the ground 702 condition. Because homogeneous regions are typically more likely to be classified correctly, the 703 accuracy estimates produced from such data would be expected to have higher accuracy than is true of 704 the study region as a whole. Consequently external validity of these data would be suspect because the 705 estimates based on the non-probability sample would not be generalizable to the target population. As 706 a second example, suppose because of convenient access the VGI data have been collected primarily at 707 locations near roads. Evaluating external validity would then require determining whether accuracy 708 near roads was equivalent to accuracy distant from roads. 
711 characteristics of the full study region. Consider the example of VGI data concentrated along roads. To

712 establish that accuracy does not vary with distance from a road, we could collect additional reference

713 data distant from roads based on a probability sampling design, and compare the accuracy estimates

714 from this sample to accuracy estimates for sample data constrained to locations near roads. But the

715 additional effort to obtain the sample data distant from roads would negate much of the value of VGI

716 for reducing the cost of accuracy assessment. That is, to definitively establish the equivalence of

717 accuracy near roads to accuracy distant from roads, we may need a large probability sample, and the

718 primary value of VGI is to reduce the cost and effort of collecting sample data.

719 Alternatively, it may be possible to cite previous studies to establish external validity. For example,

720 if previous research has demonstrated that distance from a road is not strongly related to accuracy, we

721 would have some assurance of external validity to support use of VGI data collected preferentially near

722 roads. In general, to more fully exploit the potential benefit of VGI, it may be necessary to document

723 typical features of VGI that would commonly need to be addressed to establish external validity and

724 then conduct the necessary studies to inform the decision of whether external validity is tenable.

725 Distance from road, characteristics of volunteers, and complexity of landscape are just a few examples

726 of features that might be explored to determine whether characteristics of populations (e.g., accuracy)

727 differ by these features. If in general there are no such differences, external validity of non-probability

728 sample data is supported to some degree. Developing a cohesive strategy to design and conduct such

729 studies for a broadly applicable assessment of external validity of VGI would likely require a major

730 research initiative.

731

$732 \quad 5.3 \mathrm{VGI}$ and Model-Based Inference

733 Model-based inference is not predicated on probability sampling so it is a potentially attractive 734 option for using VGI data that did not originate from a probability sampling design. Model-based 
inference requires specification of a model that relates $y_{u}$ to a set of covariates (predictors) available for the full population (Valliant et al. 2000). Developing appropriate models and evaluating the underlying assumptions may be difficult and time-consuming (Baker et al. 2013) with the difficulties exacerbated by the fact that in most surveys, numerous estimates are produced from a single sample. In the case of VGI, estimates of accuracy and area for several land-cover or land-cover change types will typically be of interest, and each of these estimates may be desired for several subregions within the target region of interest. A model will need to be developed and assumptions evaluated for all estimates as a model that works well for some estimates may not work well for others. An additional challenge to the modelbased approach is that non-probability samples may have an inherent selection bias, so a substantial risk exists that the distribution of important covariates in the sample will differ from the distribution of these covariates in the target population (Baker et al. 2013). Methods to account for preferential sampling (e.g., Diggle et al. 2010) in a model-based framework may be considered in such cases of non-probability sampling.

Numerous model-based methods can be applied to non-probability samples and evaluating the utility of model-based methods is case specific because it is difficult to ascribe general properties to these methods (Baker et al. 2013). An advantage of probability sampling and design-based inference is that a standard general approach is used to produce the complete array of estimates (see Section 2.1). Yet another challenge of model-based inference and non-probability sampling is how to define and quantify uncertainty. A widely accepted measure of precision does not exist for estimates from nonprobability samples (Baker et al. 2013, p.97), whereas the standard error (or appropriately scaled version of standard error) is generally accepted for quantifying precision of estimates in design-based inference. Clearly, some of the cost savings achieved by non-probability sampling is lost due to the more complex analyses needed to develop models and test their assumptions (Baker et al. 2013). Because model-based inference encompasses an array of methods, establishing transparency of the 
methodology is also more demanding because it is necessary to describe the specific model-based

760 approach used and the possible limitations of inference uniquely associated with that approach (Baker

761 et al. 2013, p.100).

762

\section{Discussion}

764

The increasing availability of large quantities of data obtained via non-probability sampling has garnered interest of survey methodologists in a variety of subject areas, so it is relevant to examine issues addressed in the broader survey sampling literature that go beyond just use of VGI in the remote sensing context. For example, internet surveys comprised of volunteer opt-in panels that use social media to extract information result in large quantities of data that are obtained quickly and conveniently but via a selection protocol that has no underlying probability sampling design. Review articles by Baker et al. (2013) and Elliott and Valliant (2017) provide an excellent general overview of methods and issues

771 affecting inference when using data from such non-probability samples. In the broad context of survey

772 sampling, the conventional practice of relying on design-based inference has been questioned because

773 of the tremendous increase in non-response rates. Even if a probability sampling design is

774 implemented, severe non-response will make the application of design-based inference questionable 775 (Baker et al. 2013). Fortunately, in land-cover studies non-response is generally not a major problem.

776 The availability of remote sensing platforms usually allows us to obtain the necessary observations that 777 might otherwise be very difficult if a ground visit were required. Non-response rates are typically very 778 small in accuracy assessment and area estimation applications so the dilemma of severe non-response 779 that impacts current survey practice in other fields of application is typically not a problem in land-cover 780 studies.

781 Ensuring accurate observations $\left(y_{u}\right)$ is perhaps the most challenging aspect of using VGI because it 782 depends on the volunteers to provide good quality data. Accurate interpretation of reference labels for 
783

784

785

786

787

788

789

790

791

792

793

794

795

796

797

798

799

800

801

802

803

804

805

806

land cover or land-cover change is challenging even for trained experts so label quality of VGI data needs to be scrutinized closely. A great deal of effort has been invested in improving and evaluating the quality of VGI used in land-cover studies, including the assessment of traditional quality measures such as positional, thematic or temporal accuracy (Fonte et al. 2017a), the development of new quality indicators that are applicable specifically to VGI (Meek et al. 2014; Antoniou and Skopeliti 2015; Senaratne et al. 2017), and even combinations of indicators (Bishr and Mantelas 2008; Jokar Arsanjani et al. 2015). The investment in these methods will not only yield better quality VGI data but may also contribute to improved data quality and assessment procedures applicable to reference data obtained by experts.

Baker et al. (2013) make the helpful distinction between "describers" whose purpose is to describe the population and "modelers" whose purpose is to characterize relationships between variables. Accuracy assessment and area estimation applications typically fall within the "describer" class because of the strong focus on descriptive parameters such as user's and producer's accuracies of the different classes and the area or proportion of area of the land-cover or land-cover change classes. Describers generally rely on probability sampling because of the importance of representing the target population. Elliott and Valliant (2017, p.262) provide a strong statement in support of probability sampling for descriptive objectives: “... when critical estimates of descriptive quantities such as means, quantiles or cell probabilities are required, nonprobability designs should be avoided or utilized only when it is reasonably certain that there are available covariates in both datasets related to the nonprobability selection mechanism that can be used to appropriately incorporate information from the nonprobability sample. If a sufficiently large probability sample is available for estimating descriptive statistics, methods to incorporate nonprobability data are likely not warranted."

Although design-based inference requires a probability sampling design, it is not reasonable to assert a recommendation that probability sampling must always be used. Other considerations such as 
cost and "fit for purpose "may be relevant, the latter including dimensions such as "accuracy, timeliness,

808 and accessibility" (Baker et al. 2013, p. 98). A quote from Kish (1965, pp. 28-29) extracted by Baker et al.

809 (2013, p.92) has direct bearing on this issue: "No clear rule exists for deciding exactly when probability

810 sampling is necessary, and what price should be paid for it ... Probability sampling for randomization is

811 not a dogma, but a strategy, especially for large numbers." Probability sampling offers the strong

812 advantage that it provides the basis for rigorous design-based inference, but there may be exceptional

813 cases in which fit for purpose criteria will be such that VGI from a non-probability sample will suffice.

814 While an unmistakable conclusion from our assessment of VGI for use in design-based inference is that

815 probability sampling should be used, we recognize that occasionally circumstances may exist where not

816 following this recommendation is justifiable.

VGI has great potential value within remote sensing beyond its use to produce accuracy and

818 area estimates within design-based inference. For example, VGI can greatly augment traditional sources

819 of training data used in the classification algorithms of land cover and land use maps. The exact design

820 of the training stage of a supervised classification should, however, be highly classifier-specific as

821 classifiers vary greatly in how they use the training set. While conventional statistical classifiers may

822 benefit from the use of a probability sample in the acquisition of training statistics to obtain a

823 representative and unbiased description of each class, other classifiers, such as machine learning

824 classifiers, may require only very small and distinctly non-random sample. Thus, for example, an

825 effective approach to training data acquisition for a classification by a support vector machine may be to

826 direct citizens to a small number of highly atypical training sites (Pal and Foody 2012). Classifiers also

827 vary in their sensitivity to mis-labeling of training cases (Foody et al. 2016) which may be relevant if VGI

828 is to be used.

829

Land cover data from several Geo-Wiki campaigns are now available in the openly accessible

repository Pangaea and these data could be used as training data (Fritz et al. 2017; Laso Bayas et al. 
831 2017). VGI is also useful in the development of hybrid land-cover maps, where methods such as

832 geographically weighted regression can use VGI to determine the most appropriate land cover class at a

833 given location among several existing products. Such an approach has been demonstrated in the

834 development of global land cover and forest masks (Schepaschenko et al. 2015; See et al. 2015). Finally,

835 VGI can provide a preliminary check on the accuracy of a land-cover product and guide the collection of 836 additional training data in areas where there is visual evidence of confusion between land-cover classes.

837

\section{Summary}

839

The increasing availability and quantity of VGI has generated great interest in how these data might assessment. Scientifically credible use of VGI raises many of the same issues related to inference that

842 McRoberts (2011) discussed pertaining to use of land-cover maps, stating that "...rules must be 843 rigorously followed to produce valid scientific inferences." The requirements for using VGI in rigorous 844 design-based inference are identifiable from the analysis protocol (Sec. 3.1) used to produce the area 845 and map accuracy estimates. Specifically, the estimates are derived from totals, and the Horvitz846 Thompson estimator provides an unbiased estimator of a population total if the response design 847 generates accurate observation of the attribute or measurement of interest $\left(y_{u}\right)$ and the sampling 848 design is such that the inclusion probabilities $\left(\pi_{u}\right)$ are known. If $y_{u}$ is accurate and $\pi_{u}$ is known then we 849 can produce unbiased estimators of the totals that form the basis for accuracy and area estimates. We 850 reviewed recent literature describing methods for obtaining VGI and assessing its quality (Sec. 3.2), and 851 we anticipate that ongoing research will improve reference data quality whether collected by volunteers 852 within a VGI framework or by expert interpreters.

853 The primary focus of this article has been on the sampling design issues related to using VGI in 854 design-based inference, with attention addressing three primary cases: 1) VGI data are from a 
probability sampling design; 2) VGI data from a non-probability sampling design are combined with data

856 from a probability sampling design; and 3) the only data available are VGI data from a non-probability

857 sampling design. The most direct approach to ensure that design-based inference can be invoked is to

858 specify that the VGI data will be collected at locations (sample units) selected by a probability sampling

859 design ("active VGI"). Implementing a probability sampling design ensures that the inclusion

860 probabilities $\left(\pi_{u}\right)$ for the sampled units are known and thus the corresponding estimation weights

$861\left(w_{u}=1 / \pi_{u}\right)$ required for the analysis are known. The more common situation is that the VGI data do not

862 originate from a probability sampling design. Implementing design-based inference in this situation

863 requires combining the VGI data with data obtained from a probability sampling design, and the benefit

864 of the VGI data is to reduce the standard errors of the accuracy or area estimates. Two approaches for

865 combining VGI with a probability sample are to treat the VGI as a certainty stratum (i.e., set $\pi_{u}=1$ for

866 each unit from the VGI sample) or to use the VGI to create an auxiliary variable for the population and

867 incorporate this variable in a model-assisted estimator. The certainty stratum approach is the more

868 promising of these two options particularly if a large proportion of the population is covered by VGI. For

869 land-cover studies the model-assisted estimator use of VGI likely will also incorporate maps produced

870 from remote sensing imagery.

871 If VGI data collected from a non-probability sampling design are the only data available, rigorous

872 design-based inference is not available. Estimates of accuracy and area can be produced using the same

873 estimator formulas of design-based inference by defining pseudo-estimation weights based on treating

874 the VGI as if a stratified random sample had been implemented. Estimates produced in this fashion

875 mimic the proportional representation of the feature of the population used to create the pseudo-

876 weights. However, in contrast to the case where the weights are the inverse of known inclusion

877 probabilities from a probability sampling design, the estimates based on pseudo-weights require the

878 additional step of verifying that the condition of external validity is satisfied. External validity requires 
that the population for which the VGI data are representative must have the same characteristics (e.g.,

880 model relationships) as the full population that is the target of inference. Establishing external validity is

881 often impractical so the pseudo-weight approach to using VGI from a non-probability sample will have

882 limited utility. Model-based inference is perhaps the more promising avenue for using VGI from non-

883 probability samples. Explication of model-based methods and specific example applications of accuracy

884 and area estimation (McRoberts 2006; Magnussen 2015) are needed to make model-based inference

885 more accessible to practitioners.

Invoking design-based inference as the scientific basis to support the validity of inference for

estimating area and map accuracy from sample data imposes the requirement that the sampling and

estimation protocols implemented must satisfy certain conditions. As is apparent from the methods and

discussion presented in this article, the requirement of a probability sampling design places fairly strong

restrictions on how VGI can be used in design-based inference. The methods presented in this article for

applications of accuracy assessment and area estimation.

893

894

895

896

897

898

899

900

901

902

903

904

905

906

907

908

909

910

\section{Acknowledgments}

This research was supported in part by the Portuguese Foundation for Science and Technology (FCT) under project grant UID/MULTI/00308/2013 (CF); EU-funded FP7 project CrowdLand No. 617754 and the Horizon2020 LandSense project No. 689812 (LS); Cooperative Agreement G12AC20221provided by the United States Geological Survey and NASA Carbon Monitoring System program grant NNX13AP48G (SS). We thank the reviewers for their constructive comments that led to improvements in the manuscript.

\section{References}

Antoniou, V., Morley, J., and Haklay, M. (2010). Web 2.0 geotagged photos: Assessing the spatial dimension of the phenomenon. Geomatica, 64, 99-110.

Antoniou, V., Fonte, C. C., See, L., Estima, J., Arsanjani, J. J., Lupia, F., Minghini, M., Foody, G., and Fritz, S. (2016). Investigating the feasibility of geo-tagged photographs as sources of land cover input data. ISPRS International Journal of Geo-Information, 5, 64. 
Antoniou, V., and Skopeliti, A. (2015). Measures and indicators of VGI quality: An overview. In: ISPRS Annals of the Photogrammetry, Remote Sensing and Spatial Information Sciences. Presented at the ISPRS Geospatial Week 2015, ISPRS Annals, La Grande Motte, France, pp. 345-351.

Baker, R., Brick, J. M., Bates, N. A., Battaglia, M., Couper, M. P., Dever, J. A., Gile, K. J., and Tourangeau, R. (2013). Summary report of the AAPOR Task Force on non-probability sampling. Journal of Survey Statistics and Methodology, 1, 90-105.

Bird, T. J., Bates, A. E., Lefcheck, J. S., Hill, N. A., Thomson, R. J., Edgar, G. J., Stuart-Smith, R. D., Wotherspoon, S., Krkosek, M., Stuart-Smith, J. F., Pecl, G. T., Barrett, N., and Frusher, S. (2014). Statistical solutions for error and bias in global citizen science datasets. Biological Conservation, 173, 144-154.

Bishr, M., and Mantelas, L. (2008). A trust and reputation model for filtering and classifying knowledge about urban growth. GeoJournal, 72, 229-237.

Brus, D. J., and de Gruijter, J. J. (2003). A method to combine non-probability sample data with probability sample data in estimating spatial means of environmental variables. Environmental Monitoring and Assessment, 83, 303-317.

Card, D. H. (1982). Using known map category marginal frequencies to improve estimates of thematic map accuracy. Photogrammetric Engineering \& Remote Sensing, 48, 431-439.

Ciepłuch, B., Jacob, R., Mooney, P., and Winstanley, A. (2010). Comparison of the accuracy of OpenStreetMap for Ireland with Google Maps and Bing Maps. Proceedings of the Ninth International Symposium on Spatial Accuracy Assessment in Natural Resources and Environmental Sciences, 20-23 July, 2010, 337.

Clark, M.L. and Aide, T.M. (2011). Virtual Interpretation of Earth Web-Interface Tool (VIEW-IT) for collecting land-use/land-cover reference data. Remote Sensing, 3, 601-620.

Cochran, W. G. (1977). Sampling Techniques, 3rd ed. (New York: John Wiley \& Sons).

De Gruijter, J. J., and Ter Braak, C. J. F. (1990). Model-free estimation from spatial samples: A reappraisal of classical sampling theory. Mathematical Geology, 22, 407-415.

Dever, J. A., Rafferty, A., and Valliant, R. (2008). Internet surveys: Can statistical adjustments eliminate coverage bias? Survey Research Methods, 2, 47-62.

Dever, J. A., and Valliant, R. (2014). Estimation with non-probability surveys and the question of external validity. Proceedings of Statistics Canada Symposium 2014, 8 pp. 
950

951

952

953

954

955

956

957

958

959

960

961

962

963

964

965

966

967

968

969

970

971

972

973

974

975

976

977

978

979

980

981

982

983

984

985

986

987

988

Deville, J. C., and Särndal, C. E. (1992). Calibration estimators in survey sampling. Journal of the American Statistical Association, 87, 376-382.

Diggle, P., Menezes, R., and Su, T. (2010). Geostatistical inference under preferential sampling. Applied Statistics, 59, 191-232.

Diggle, P. J., Tawn, J. A., and Moyeed, R. A. (1998). Model-based geostatistics. Applied Statistics, 47, 299350.

Elliott, M. R., and Valliant, R. (2017). Inference for nonprobability samples. Statistical Science, 32, 249264.

Elwood, S., Goodchild, M.F., and Sui, D. (2013). Prospects for VGI research and the emerging fourth paradigm. Crowdsourcing geographic knowledge: Volunteered geographic information (VGI) in theory and practice, Springer, Netherlands, pp. 361-375.

Fonte, C. C., Bastin, L., See, L., Foody, G., and Lupia, F. (2015). Usability of VGI for validation of land cover maps. International Journal of Geographical Information Science, 29, 1269-1291.

Fonte, C.C., Antoniou, V., Bastin, L., Estima, J., Arsanjani, J.J., Laso-Bayas, J.-C., See, L., and Vatseva, R. (2017a). Assessing VGI data quality, in: Foody, G.M., See, L., Fritz, S., Fonte, C.C., Mooney, P., OlteanuRaimond, A.-M., Antoniou, V. (Eds.), Mapping and the Citizen Sensor. Ubiquity Press, London, UK, pp. 137-164.

Fonte, C. C., Patriarca, J. A., Minghini, M., Antoniou, V., See, L., and Brovelli, M. A. (2017b). Using OpenStreetMap to create land use and land cover maps: Development of an application. In: Volunteered Geographic Information and the Future of Geospatial Data (Ed.: Campelo, C. E. C., Bertolotto, M., and Corcoran, P.). IGI Global. DOI: 10.4018/978-1-5225-2446-5.ch007

Foody, G. M. (2009). The impact of imperfect ground reference data on the accuracy of land cover change estimation. International Journal of Remote Sensing, 30, 3275-3281.

Foody, G.M. (2010). Assessing the accuracy of land cover change with imperfect ground reference data. Remote Sensing of Environment, 114, 2271-2285.

Foody, G.M., Pal, M., Rocchini, D., Garzon-Lopez, C.X. and Bastin, L. (2016). The sensitivity of mapping methods to reference data quality: Training supervised image classifications with imperfect reference data. ISPRS International Journal of Geo-Information, 5(11), p.199.

Foody, G. M., See, L., Fritz, S., Van der Velde, M., Perger, C., Schill, C., and Boyd, D. S. (2013). Assessing the accuracy of volunteered geographic information arising from multiple contributors to an internet based collaborative project. Transactions in GIS, 17, 847-860. 
Foody, G.M., See, L., Fritz, S., Velde, M. van der, Perger, C., Schill, C., Boyd, D.S., and Comber, A. (2015). Accurate attribute mapping from Volunteered Geographic Information: Issues of volunteer quantity and quality. The Cartographic Journal, 52, 336-344.

Fritz, S., McCallum, I., Schill, C., Perger, C., See, L., Schepaschenko, D., van der Velde, M., Kraxner, F., and Obersteiner, M. (2012). Geo-Wiki: An online platform for improving global land cover. Environmental Modelling \& Software, 31, 110-123.

Fritz, S., See, L., van der Velde, M., Nalepa, R.A., Perger, C., Schill, C., McCallum, I., Schepaschenko, D., Kraxner, F., Cai, X., Zhang, X., Ortner, S., Hazarika, R., Cipriani, A., Di Bella, C., Rabia, A.H., Garcia, A., Vakolyuk, M., Singha, K., Beget, M.E., Erasmi, S., Albrecht, F., Shaw, B., and Obersteiner, M. (2013). Downgrading recent estimates of land available for biofuel production. Environmental Science and Technology, 47, 1688-1694.

Fritz, S., See, L., Perger, C., McCallum, I., Schill, C., Schepaschenko, D., Duerauer, M., Karner, M., Dresel, C., Laso Bayas, J.C., Lesiv, M., Moorthy, I., Salk, C., Danylo, O., Sturn, T., Albrecht, F., You, L., Kraxner, F. and Obersteiner, M. (2017). A global dataset of crowdsourced land cover and land use reference data. Scientific Data, 4:170075. doi: 10.1038/sdata.2017.75.

Gallego, F. J. (2004). Remote sensing and land cover area estimation. International Journal of Remote Sensing, 25, 3019-3047.

Girres, J.-F., and Touya, G. (2010). Quality assessment of the French OpenStreetMap dataset. Transactions in GIS, 14, 435-459.

Goodchild, M. F. (2007). Citizens as sensors: The world of volunteered geography. GeoJournal, 69, 211221.

Gregoire, T. G. (1998). Design-based and model-based inference in survey sampling: appreciating the difference. Canadian Journal of Forest Research, 28, 1429-1447.

Haklay, M. (2010). How good is volunteered geographical information? A comparative study of OpenStreetMap and Ordnance Survey datasets. Environment and Planning B: Planning and Design, 37,682-703.

Horvitz, D. G., and Thompson, D. J. (1952). A generalization of sampling without replacement from a finite universe. Journal of the American Statistical Association, 47, 663-685.

Hou, D., Chen, J., Wu, H., Li, S., Chen, F., and Zhang, W. (2015). Active collection of land cover sample data from geo-tagged web texts. Remote Sensing, 7, 5805-5827.

Isaaks, E. H., and Srivastava, R. M. (1989). An Introduction to Applied Geostatistics, Oxford University Press, New York. 
Iwao, K., Nishida, K., Kinoshita, T., and Yamagata, Y. (2006). Validating land cover maps with Degree Confluence Project information. Geophysical Research Letters, 33, L23404, doi:10.1029/2006GL027768.

Jokar Arsanjani, J. and Bakillah, M. (2015). Understanding the potential relationship between the socioeconomic variables and contributions to OpenStreetMap. International Journal of Digital Earth, 8, 861876.

Kish, L. (1965). Survey Sampling. Wiley, New York.

Laso Bayas, J.C., Lesiv, M., Waldner, F., Schucknecht, A., Duerauer, M., See, L. et al. (2017). A global reference database of crowdsourced cropland data collected using the Geo-Wiki platform. Scientific Data. 4: 170136. doi:10.1038/sdata.2017.136.

Laso Bayas, J.-C., See, L., Fritz, S., Sturn, T., Perger, C., Duerauer, M., Karner, M., Moorthy, I., Schepaschenko, D., Domian, D., and McCallum, I. (2017). Crowdsourcing in-situ data on land cover and land use using gamification and mobile technology. Remote Sensing 8(11), 905. doi:10.3390/rs8110905.

Loosveldt, G., and Sonck, N. (2008). An evaluation of the weighting procedures for an online access panel survey. Survey Research Methods, 2, 93-105.

Magnussen, S. (2015). Arguments for a model-dependent inference? Forestry, 88, 317-325.

Martino, L., Palmieri, A., and Gallego, J. (2009). Use of auxiliary information in the sampling strategy of a European area frame agro-environmental survey. Available from: http://ec.europa.eu/eurostat/documents/205002/769457/LUCAS2009_S2-Sampling_20090000.pdf. Eurostat.

McRoberts, R. E. (2006). A model-based approach to estimating forest area. Remote Sensing of Environment, 103, 56-66.

McRoberts, R. E. (2010). Probability- and model-based approaches to inference for proportion forest using satellite imagery as ancillary data. Remote Sensing of Environment, 114, 1017-1025.

McRoberts, R. E. (2011). Satellite image-based maps: Scientific inference or pretty pictures? Remote Sensing of Environment, 115, 715-724.

Meek, S., Jackson, M.J., and Leibovici, D.G. (2014). A flexible framework for assessing the quality of crowdsourced data. In: Huerta, J., Schade, S., and Granell, C. (Eds.), Connecting a Digital Europe through Location and Place: Proceedings of the AGILE'2014 International Conference on Geographic Information Science. Presented at the AGILE'2014 International Conference on Geographic Information Science, AGILE Digital Editions, Castellón, Spain.

Meek, S., Jackson, M., and Leibovici, D.G. (2016). A BPMN solution for chaining OGC services to quality assure location-based crowdsourced data. Computers \& Geosciences, 87, 76-83. 
Mullen, W. F., Jackson, S. P., Croitoru, A., Crooks, A., Stefanidis, A., and Agouris, P. (2014). Assessing the impact of demographic characteristics on spatial error in volunteered geographic information features. GeoJournal, 80, 587-605.

Olofsson, P., Foody, G. M., Stehman, S. V., and Woodcock, C. E. (2013). Making better use of accuracy data in land change studies: Estimating accuracy and area and quantifying uncertainty using stratified estimation. Remote Sensing of Environment, 129, 122-131.

Olofsson, P., Foody, G. M., Herold, M., Stehman, S. V., Woodcock, C. E., and Wulder, M. A. (2014). Good practices for estimating area and assessing accuracy of land change. Remote Sensing of Environment, $148,42-57$.

Overton, J. M., Young, T. C., and Overton, W. S. (1993). Using 'found' data to augment a probability sample: Procedure and case study. Environmental Monitoring and Assessment, 26, 65-83.

Pal, M. and Foody, G.M. (2012). Evaluation of SVM, RVM and SMLR for accurate image classification with limited ground data. IEEE Journal of Selected Topics in Applied Earth Observations and Remote Sensing, 5, 1344-1355.

Sannier, C., McRoberts, R. E., Fichet, L.-V., \& Makaga, E. M. K. (2014). Using the regression estimator with Landsat data to estimate proportion forest cover and net proportion deforestation in Gabon. Remote Sensing of Environment, 151, 138-148.

Särndal, C. E., Swensson, B., and Wretman, J. (1992). Model-Assisted Survey Sampling. Springer-Verlag, New York.

Schepaschenko, D., See, L., Lesiv, M., McCallum, I., Fritz, S., Salk, C., Moltchanova, E., Perger, C., Shchepashchenko, M., Shvidenko, A., Kovalevskyi, S., Gilitukha, D., Albrecht, F., Kraxner, F., Bun, A., Maksyutov, S., Sokolov, A., Dürauer, M., Obersteiner, M., Karminov, V., and Ontikov, P. (2015). Development of a global hybrid forest mask through the synergy of remote sensing, crowdsourcing and FAO statistics. Remote Sensing of Environment, 162, 208-220.

See, L., Comber, A., Salk, C., Fritz, S., van der Velde, M., Perger, C., Schill, C., McCallum, I., Kraxner, F., and Obersteiner, M. (2013). Comparing the quality of crowdsourced data contributed by expert and non-experts. PLOS ONE 8, e69958. doi:10.1371/journal.pone.0069958

See, L., Fritz, S., Perger, C., Schill, C., McCallum, I., Schepaschenko, D., Duerauer, M., Sturn, T., Karner, M., Kraxner, F., and Obersteiner, M. (2015). Harnessing the power of volunteers, the internet and Google Earth to collect and validate global spatial information using Geo-Wiki. Technological Forecasting and Social Change 98, 324-335.

See, L., Mooney, P., Foody, G., Bastin, L., Comber, A., Estima, J., Fritz, S., Kerle, N., Jiang, B., Laakso, M., Liu, H.-Y., Milčinski, G., Nikšič, M., Painho, M., Pődör, A., Olteanu-Raimond, A.-M., and Rutzinger, M. (2016). Crowdsourcing, Citizen Science or Volunteered Geographic Information? The current state of 
1098

1099

1100

1101

1102

1103

1104

1105

1106

1107

1108

1109

1110

1111

1112

1113

1114

1115

1116

1117

1118

1119

1120

1121

1122

1123

1124

1125

1126

1127

1128

1129

1130

1131

1132

crowdsourced geographic information. ISPRS International Journal of Geo-Information, 5(5), 55. doi: 10.3390/ijgi5050055

See, L., Laso Bayas, J.C., Schepaschenko, D., Perger, C., Dresel, C., Maus, V., Salk, C., Weichselbaum, J. Lesiv, M., McCallum, I., Moorthy, I. and Fritz, S. (2017). LACO-Wiki: A new online land cover validation tool demonstrated using GlobeLand30 for Kenya. Remote Sensing 9(7), 754; doi:10.3390/rs9070754.

Senaratne, H., Mobasheri, A., Ali, A.L., Capineri, C., and and Haklay, M. (2017). A review of volunteered geographic information quality assessment methods. International Journal of Geographical Information Science, 31, 139-167.

Stehman, S. V. (1999). Basic probability sampling designs for thematic map accuracy assessment. International Journal of Remote Sensing, 20, 2423-2441.

Stehman, S. V. (2000). Practical implications of design-based sampling inference for thematic map accuracy assessment. Remote Sensing of Environment, 72, 35-45.

Stehman, S. V. (2009). Model-assisted estimation as a unifying framework for estimating the area of land cover and land-cover change from remote sensing. Remote Sensing of Environment, 113, 2455-2462.

Stehman, S. V. (2013). Estimating area from an accuracy assessment error matrix. Remote Sensing of Environment, 132, 202-211.

Stehman, S. V., and Czaplewski, R. L. (1998). Design and analysis for thematic map accuracy assessment: Fundamental principles. Remote Sensing of Environment, 64, 331-344.

Strahler, A. H., Boschetti, L., Foody, G. M., Friedl, M. A., Hansen, M. C., Herold, M., Mayaux, P., Morisette, J. T., Stehman, S. V., and Woodcock, C. E. (2006). Global land cover validation:

Recommendations for evaluation and accuracy assessment of global land cover maps, EUR 22156 EN DG, Office for Official Publications of the European Communities, Luxembourg, 48 pp.

Tsendbazar, N.E., de Bruin, S., and Herold, M. (2015). Assessing global land cover reference datasets for different user communities. ISPRS Journal of Photogrammetry and Remote Sensing, 103, 93-114.

Valliant, R. (2013). Comment on "Summary report of the AAPOR Task Force on non-probability sampling." Journal of Survey Statistics and Methodology, 1, 105-111.

Valliant, R., and Dever, J. A. (2011). Estimating propensity adjustments for volunteer web surveys. Sociological Methods \& Research, 40, 105-137.

Valliant, R., Dorfman, A. H., and Royall, R. M. (2000). Finite Population Sampling and Inference: A Prediction Approach, John Wiley \& Sons, Inc., New York. 
1133

1134 LIST OF FIGURE CAPTIONS

1135 Figure 1.

1136 Figure 2.

1137 Figure 3.

1138 